\title{
Karstphänomene in den bolivianischen Anden
}

\section{Problemstellung}

In den bolivianischen Anden sind verkarstungsfähige Gesteine, Kalk, Dolomit und Gips, nicht häufig anzutreffen. Sie sind durch ihre Löslichkeit und die dadurch gegebene Formenwelt gekennzeichnet: Karstlandschaften mit unterirdischer Entwässerung. Allerdings sind sie für den Andenraum, besonders auch in Bolivien, erst wenig untersucht worden. Den ersten Überblick zu Karsterscheinungen in den Anden gibt KINZL (1951). Darin erwähnt er vor allem Beobachtungen aus Peru, daneben aber auch aus Venezuela durch vON HUMBOLDT (1861) und aus Kolumbien durch HETTNER (1881, zit. in KINZL 1951, S. 52).

Bei Feldarbeiten 1977 und 1981 zur pollenanalytischen Erforschung der postglazialen Klimageschichte versuchte ich, einige die Karstlandschaften betreffende Probleme $\mathrm{zu}$ erfassen und erste Abklärungen $\mathrm{zu}$ erbringen:

1. Welche Oberflächenformen treten hier auf?

2. Welches sind die klimatischen Bedingungen, und wie weit reichen Karstlandschaften in den Bereich von Trocken- und Kältewüsten hinein?

3. Wie groß ist der petrographische Spielraum?

4. Ist eine Altersdatierung der Verkarstung möglich?

Diese Fragen bzw. Problemkreise liegen den folgenden vier Kapiteln zugrunde. Da arbeitsmethodisch die Bestandesaufnahme an erster Stelle steht, ist zu Beginn ein Katalog der angetroffenen Karstformen aufgeführt'.

\section{Formenschatz}

Die gefundenen Karstformen sind kartographisch nur so weit festgehalten worden, als dies eine Lokalisierung im Überblick erfordert. So zeigt denn Fig. 1 die im Text erwähnten Lokalitäten, welche sich alle in der bolivianischen Zentralkordillere befinden. Ihr höhenmäßiges Verbreitungsgebiet erstreckt sich von $2400 \mathrm{~m}$ am Andenosthang bei Sorata und $2800 \mathrm{~m}$ im interandinen Becken von Cochabamba bis $4600 \mathrm{~m}$ im Grenzgebiet von Bolivien und Peru. Hauptsächlich wurden Karstformen in den Höhenstufen der Puna (3700-4500 m) und der Puna brava (d. h. der subnivalen Stufe oberhalb ca. $4500 \mathrm{~m}$ ) angetroffen. Frostschutt und Moränen traten oft damit vergesellschaftet auf.

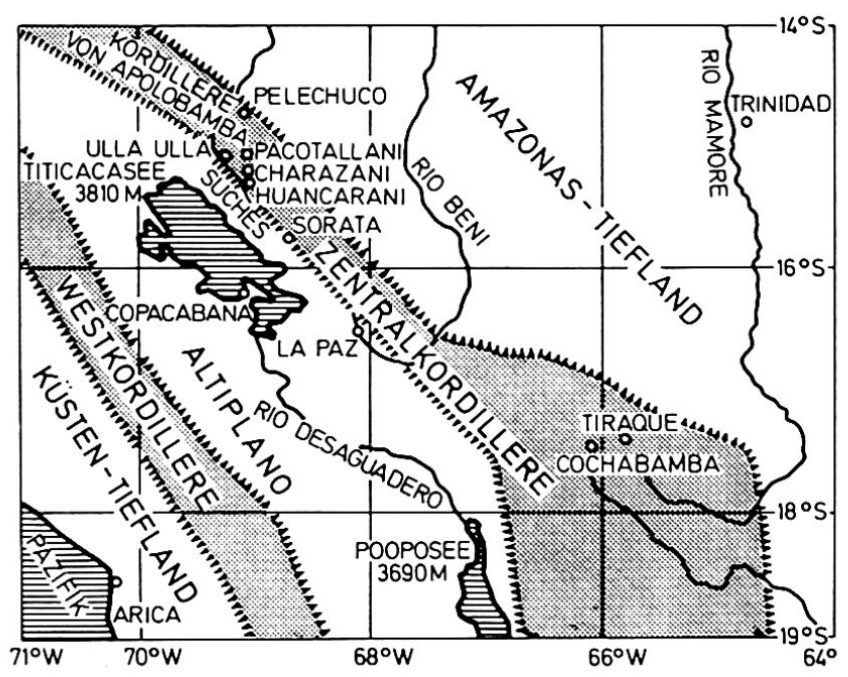

Fig.1 Überblickskarte zum bolivianischen Untersuchungsgebiet. Ein Punktraster kennzeichnet die beiden Hauptketten der Anden, die das Altiplano säumen.

Die häufigsten Zeugen von Karstprozessen sind Karren. In Abb. 1 und 2 sind zahlreiche dieser Kleinformen erkennbar, welche einen Rundhöcker am Río Pacotallani ( $15^{\circ} 3^{\prime} 40^{\prime \prime} \mathrm{S} / 69^{\circ} 7^{\prime} \mathrm{W}, 4430 \mathrm{~m}$ ) in Gefällsrichtung überprägt haben (v.a. Rinnenkarren) oder längs Klüften durchziehen (Kluftkarren). Die Korrosion hat die Karren so stark erweitert, daß die Oberfläche in viele Rechtecke und Trapeze unterteilt worden ist. Die Rinnenkarren sind bis ca. $50 \mathrm{~cm}$ tief, die Kluftkarren sogar oft über $1 \mathrm{~m}$, und sie weisen als Füllung einen humosen bis lehmigen, rotbraunen Boden und krautige Vegetation auf.

Ein weiteres Vorkommen von Rinnenkarren liegt im Muschelkalkgebiet zwischen Charazani und Huancarani ( $\left.15^{\circ} 14^{\prime} \mathrm{S} / 69^{\circ} 2^{\prime} \mathrm{W}, 4570 \mathrm{~m}\right)$. Hier sind die Karren oft moosbedeckt und leicht gerundet, also eventuell Rundkarren. Ähnlich ausgebildete Karren sind auch am östlichen Rand der Halbinsel Copacabana am Titicacasee angetroffen worden $\left(16^{\circ} 3^{\prime} \mathrm{S} / 69^{\circ} 0^{\prime} \mathrm{W}\right.$, $4000-4050 \mathrm{~m})$. Gelegentlich tragen diese gegen NE

Kurt Graf, PD, Dr., Geographisches Institut, Universität Zürich-Irchel, $\mathrm{CH}-8057$ Zürich 


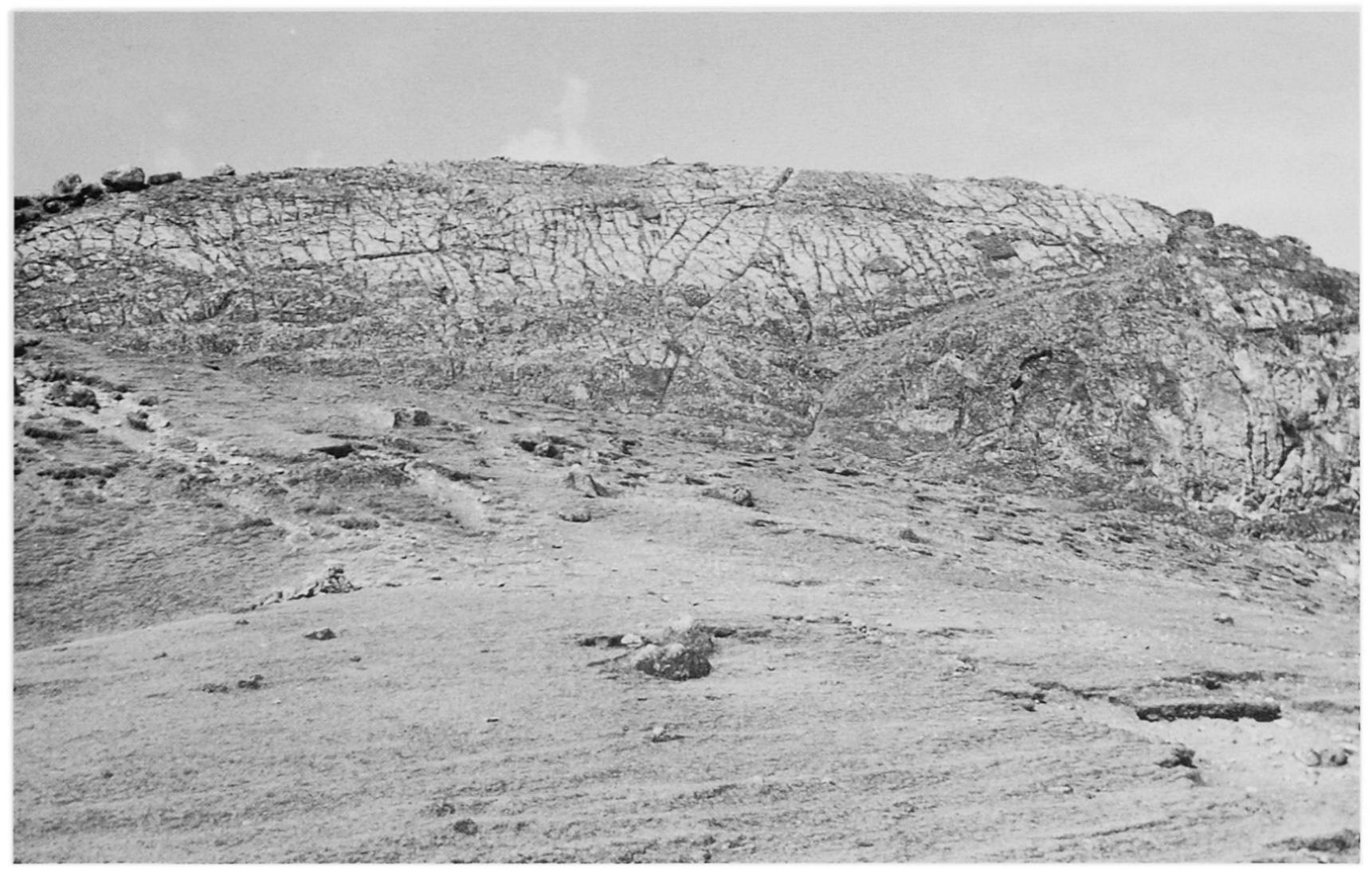

Abb. 1 Ein $20 \mathrm{~m}$ hoher Rundhöcker bei Pacotallani ( $15^{\circ} 3^{\prime} 40^{\prime \prime} \mathrm{S} / 69^{\circ} 7^{\prime} \mathrm{W}, 4430 \mathrm{~m}$ ) ist von Rinnen- und Kluftkarren gemustert. Der letzteiszeitliche Gletscher stieß von links (von N) her vor und ließ im Vordergrund Moränenmaterial zurück.

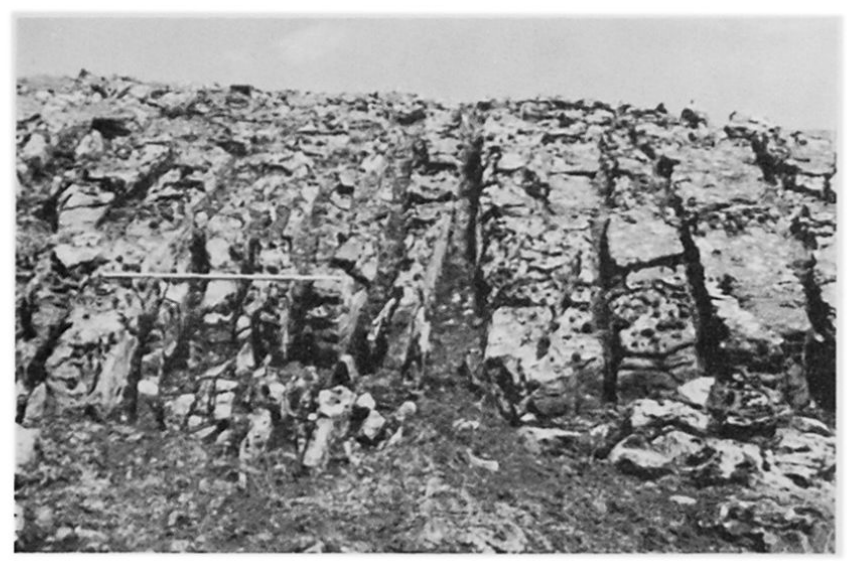

Abb. 2 Detailaufnahme zu Abb.1. Ein Doppelmeter ist in Streichrichtung des Hanges ausgelegt. Senkrecht dazu verlaufen 30-50 cm tiefe Rinnenkarren.

geneigten Flächen zwischen den Rinnen noch eine weitere Karrenform, nämlich Wurzelkarren (Abb. 3).

Es sind kleine Furchen, die nur wenige $\mathrm{cm}$ lang werden und in zufälliger Anordnung wirr ihre Richtung wechseln. Sie entstehen am Kontakt von Rhizosphäre und Kalkfels unter dem Einfluß von Wurzelsäuren, möglicherweise auch durch Dissimilations-

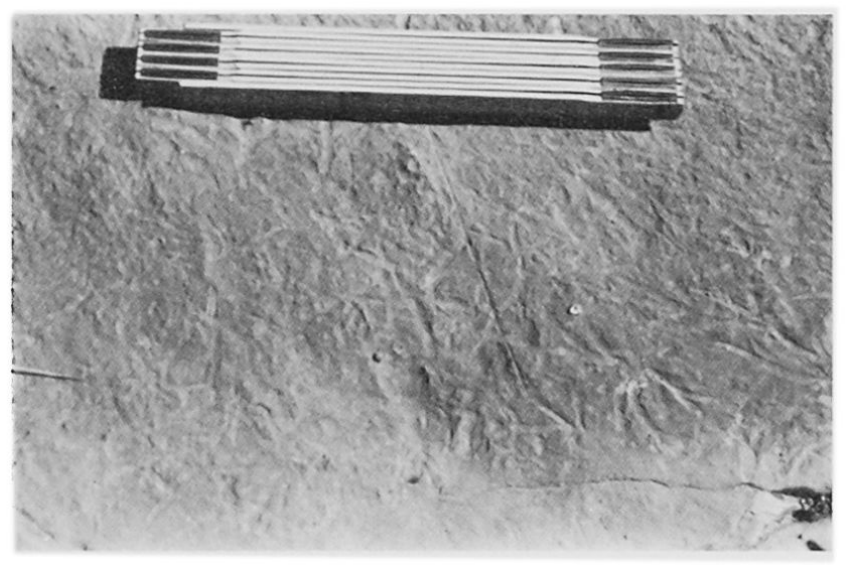

Abb. 3 Wurzelkarren auf $15^{\circ}$ geneigter Kalkunterlage am Ostrand der Halbinsel Copacabana (16 $6^{\circ} 3^{\prime} \mathrm{S} / 69^{\circ} 0^{\prime} \mathrm{W}$, $4020 \mathrm{~m}$ ). Im weiteren ist in der Bildmitte eine Strukturkarre erkennbar und am rechten unteren Bildrand eine undeutlich ausgebildete Mäanderkarre.

kohlensäure. Aufgrund der flächenhaften Ausbildung sind wohl Polsterpflanzen an der Genese beteiligt gewesen. Die Felsfläche mag bis vor wenigen Jahrzehnten von dünnen Bodenhorizonten mit reichlich Wurzelwerk besetzt gewesen sein, die dann durch Beweidung oder infolge Klimaänderungen ${ }^{2}$ zerstört worden sind. Nach der Entblössung des Kalkunter- 
grundes hat die Korrosion eingesetzt und die Wurzelkarren seither etwas verwischt. Abb. 3 zeigt auch eine Strukturkarre, die von der Mitte des Klappmeters schräg nach rechts unten verläuft. Sie weist V-förmigen Querschnitt auf.

Am gleichen Abhang zum Titicacasee hin zeichnen sich die Karren noch durch weitere Besonderheiten aus. An flacheren Abschnitten treten bei $15-25^{\circ}$ Gefälle bloß $1-5 \mathrm{~cm}$ tiefe Kluft- und Rinnenkarren (und z. T. Wurzelkarren) auf. Besonders klein sind sie in konkaven Hangpartien, wo das Wasser eher langsam abfließt. Umgekehrt findet man größere Rinnen- und Rundkarren dort, wo der Hang mit $40-50^{\circ}$ gegen NE abfällt und konvex ist. Auch $3 \mathrm{~cm}$ breite Kluftkarren können hier $10 \mathrm{~cm}$ tief hinabreichen. An diesen steilen Partien wachsen oft Moospolster und Büschelgräser in den Karren. Die Dynamik der Bildungsprozesse kann anhand dieser Einzelbeobachtungen nicht erklärt werden. Es scheint aber, daß diese stellenweise gerundeten Karren ursprünglich bodenbedeckt waren und erst sekundär entblößt wurden. Jedenfalls bestätigt sich hier, daß für die Art und Intensität der Karrenformung das Ausgangsrelief und das Abflußregime eine wichtige Rolle spielen (BÖGLI 1951, S. 195-197) ${ }^{3}$.

Im größeren Maßstab kennzeichnen die Dolinen verkarstete Flächen. Zahlreich finden sie sich nord-

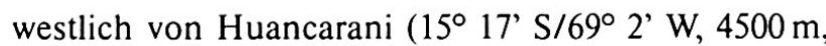
Abb.4; s. auch PEREZ 1972, S.32). Auch fast $30 \mathrm{~km}$ südlich davon hat man schöne Dolinen vor sich, wo die Straße auf ca. $4200 \mathrm{~m}$ zum Río Suches hin abzweigt. Oftmals verrät ihre lineare Anordnung die Entstehung auf Verwerfungen bzw. tektonischen Linien, wo das Wasser frühzeitig hat abfließen können. Als trichterförmige Mulden erreichen sie hier Tiefen bis $5 \mathrm{~m}$ und Durchmesser von $15 \mathrm{~m}$. Im Kalk ist die Vegetation geschlossen, hingegen im Gips (Abb.4) durch sekundäre Erosion und Rutschungen

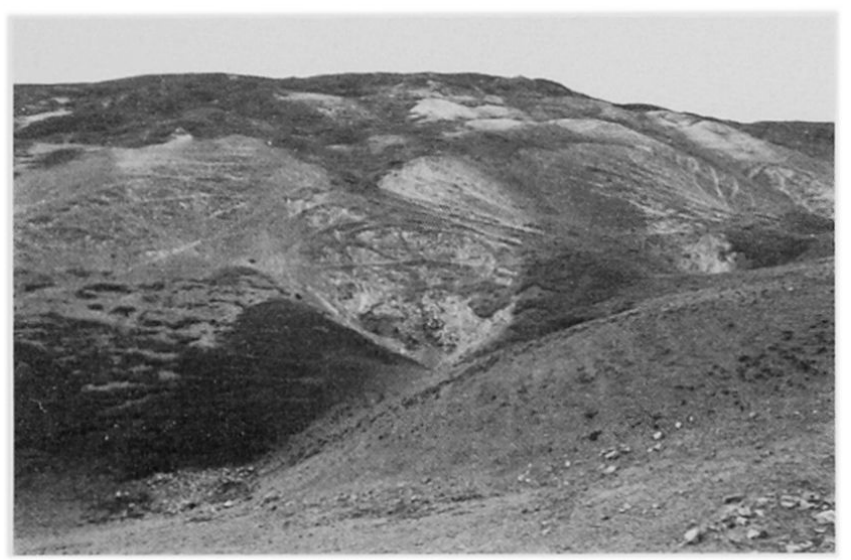

Abb. 4 Diese drei besonders tiefen Dolinen sind bei Huancarani aneinandergereiht $\left(15^{\circ} 17^{\prime} \mathrm{S} / 69^{\circ} 2^{\prime} \mathrm{W}, 4500 \mathrm{~m}\right)$. Weil mit Gips ein weiches Gestein ansteht, ist die Vegetationsdecke durch Rutschungen beeinträchtigt worden. gelichtet oder fast gänzlich zerstört ${ }^{4}$. Ein ähnliches Bild präsentiert sich wenig östlich von Pacotallani $\left(15^{\circ}\right.$ 4' S/69 7' W, 4350-4400 m). Hier sind im Gips bis $4 \mathrm{~m}$ tiefe Dolinen vorhanden, Kalk ist hingegen lediglich mit Karren übersät. In der näheren Umgebung von Huancarani haben sich zahlreiche Dolinen zu einem weiten Dolinenfeld vereinigt $\left(15^{\circ} 18^{\prime} \mathrm{S} / 69^{\circ} 0^{\prime} \mathrm{W}\right.$, ca. $4300 \mathrm{~m}$ ). Abb. 5 zeigt diese Landschaft, wo wiederum Kalk und Gips am Aufbau beteiligt sind.

Als weitere Erscheinungen im Karstgebiet Nordboliviens seien Hangquellen erwähnt. Sie treten zahlreich im Tal von Charazani und den oberen Talabschlüssen im angrenzenden Hochgebirge auf. Meistens sind es Schichtgrenzquellen am Übergang von Kalk zu einer undurchlässigen Unterlage aus Tonschiefer. Ob es sich teilweise um Karstquellen handelt, kann nicht eindeutig belegt werden, da keine Tracer eingesetzt worden sind. Immerhin sprechen einige Indizien dafür, daß es sich m.E. um Karsterscheinungen handeln dürfte (s. Kap. 4.1).

Die Höhle von San Pedro bei Sorata ist eine speläologische Besonderheit ( $\left.15^{\circ} 44^{\prime} \mathrm{S} / 68^{\circ} 41^{\prime} \mathrm{W}, 2400 \mathrm{~m}\right)$. Sie ist eine reine Gipshöhle und erreicht die ungewöhnlichen Maße von rund $10 \mathrm{~m}$ Höhe und $20 \mathrm{~m}$ Breite. Wahrscheinlich ist sie auf einer Kluft angelegt. Der Zutritt ist am Eingang durch Inkasion erschwert, doch nachher öffnet sie sich und gibt eine Gipskiesfläche frei. Tunnelartig führt der Gang nach etwa $50 \mathrm{~m} \mathrm{zu}$ einem unterirdischen See, der in weitem Bogen hinter einer Kulisse verschwindet. Gipshöhlen sind von NUÑEZ JIMENEZ (1969, S. 33) auch auf Kuba gefunden worden.

In der Literatur über Lateinamerika sind v. a. Karstformen aus heißen, feuchttropischen Regionen beschrieben worden, und ihren Formenkreis kennzeichnen deutlich größere, schönere und auffallend andere Formen als in Bolivien. So stehen also die Karstlandschaften Boliviens (und Perus, S. KINZL 1951) in

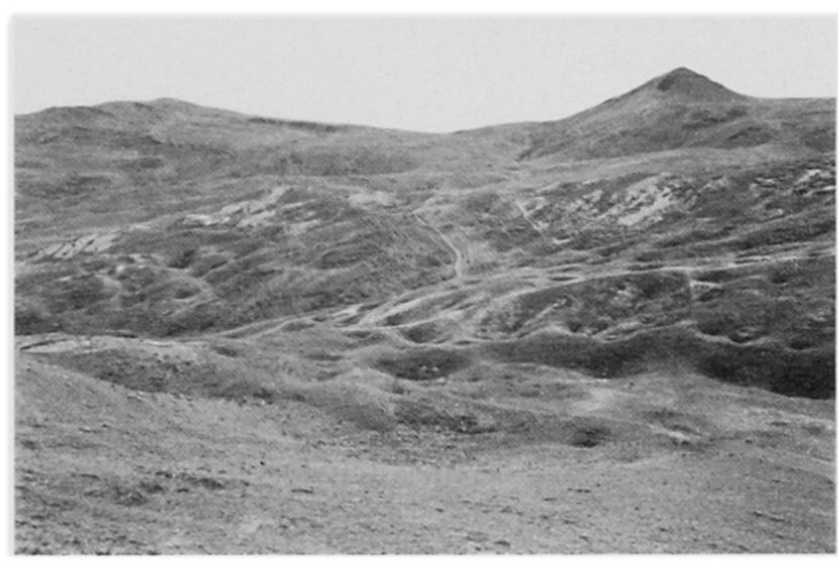

Abb. 5 Ein Dolinenfeld bei Huancarani (15० $18^{\prime} \mathrm{S} / 69^{\circ} \mathrm{O}^{\prime} \mathrm{W}$ ) hat sich im engmaschigen Wechselspiel von Kalk und Gips ausgebildet. Da dieser ca. $4300 \mathrm{~m}$ hoch gelegene Talabschnitt von Lamas und Alpacas stark beweidet wird, können sich Schäden an der Grasnarbe einstellen. 
deutlichem Gegensatz zu den Karsttypen Mittelamerikas und Mexicos. Wichtig ist für Mexico und die Großen Antillen der Kegelkarst mit seinen verschieden weit entwickelten Varietäten (LEHMANN 1954; GeRstenhauer 1966, S. 23f.). Dazu gehören auch Karstrandebenen und Interior Valleys (VERSEY 1972, S. 462f.). Poljen und ähnliche Großformen sind ebenfalls häufig beschrieben worden, so aus Mexico (WENZENS 1973, S. 58), Kuba (LEHMANN 1954, S. 130/131), Jamaica (sweeting 1972) und Puerto Rico (MONRoE 1960; MIOTKe 1973). Aus Yucatán sind tiefe Schächte bekanntgeworden, sog. "Cenotes» (CORBEL 1959, S. 5). Sie reichen im dortigen Kalkplateau 8-54 m tief hinab in die unterirdischen Karstgrundwasserseen (вöGLI, mdl. Mitt.). Damit weist sich das feuchttropische Lateinamerika durch einen reichen Karstformenschatz aus. Daneben erscheint der Karst unseres Untersuchungsgebiets ärmlich, was natürlich teilweise klimatisch begründet ist, liegt es doch in einem vorwiegend wechselfeuchten Klimabereich.

\section{Klima}

Das klimatische Spektrum des Untersuchungsraums umfaßt Köppens Klimatypen ET (Tundrenklima), Cw (wintertrockenes gemäßigtes Klima) und BS (Steppenklima). Aus Fig. 2 sind diese relativ trockenen, kalten Bedingungen ablesbar. Die Niederschläge beschränken sich vorwiegend auf Dezember bis März und erzielen Jahressummen von $49 \mathrm{~cm}$ (Ulla Ulla), $55 \mathrm{~cm}$ (Cochabamba), $61 \mathrm{~cm}$ (Tiraque), $79 \mathrm{~cm}$ (Sorata), $86 \mathrm{~cm}$ (Pelechuco) bis $102 \mathrm{~cm}$ (Copacabana). Die Anzahl der humiden Monate beträgt 4 bis 8 , was semiarides bis semihumides Klima bedeutet (LAUer 1960, Abb. 14). Karstformen fehlen also in Bolivien lediglich im ariden Klimabereich (BW nach KÖPPEN). Der weitgehend ausbleibende Niederschlag ist generell für Karstprozesse ungünstig. Für aride Kalkgebiete im Süden des Landes wäre aber durchaus denkbar, daß Kondenswasser korrosiv wirkte und auf der Unterseite flacher Steine Miniaturkarren bildete. Diese Feuchtigkeit ist allerdings ein Wasserfilm von sehr geringer
ULLA ULLA

$15^{\circ} 4^{\prime} \mathrm{S} / 69^{\circ} 15^{\prime} \mathrm{W}, 4460 \mathrm{~m}$

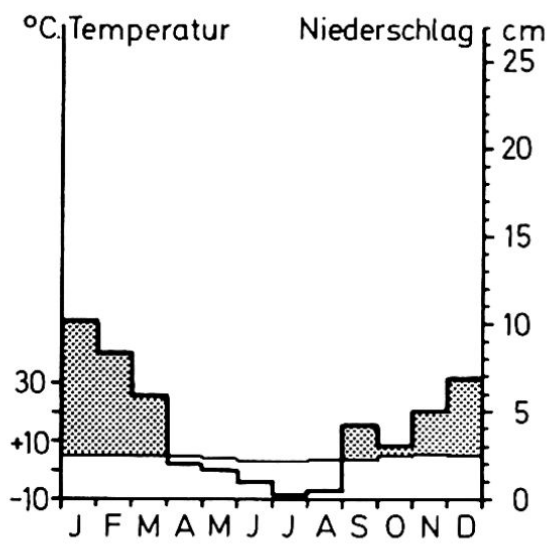

PELECHUCO

$14^{\circ} 43^{\prime} \mathrm{S} / 69^{\circ} 5^{\prime} \mathrm{W}, 3610 \mathrm{~m}$

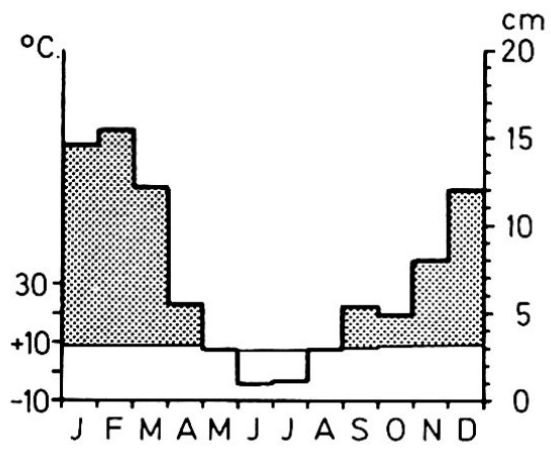

COPACABANA

$16^{\circ} 10^{\prime} \mathrm{S} / 69^{\circ} 5^{\prime} \mathrm{W}, 3840 \mathrm{~m}$



SORATA

$15^{\circ} 44^{\prime}$ S / 68 $40^{\prime} \mathrm{W}, 2700 \mathrm{~m}$

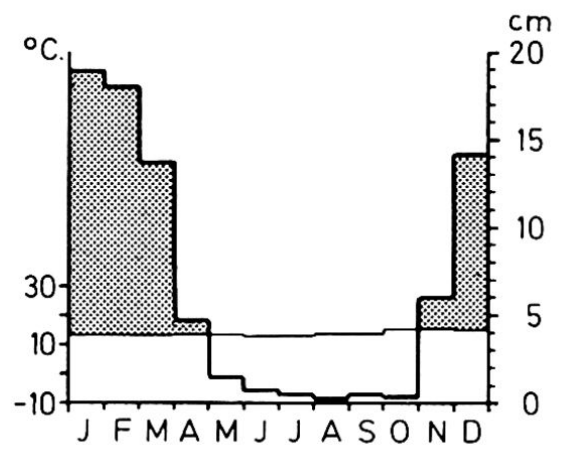

TIRAQUE

$17^{\circ} 24^{\prime} \mathrm{S} / 65^{\circ} 44^{\prime} 30^{\prime \prime} \mathrm{W}, 3220 \mathrm{~m}$

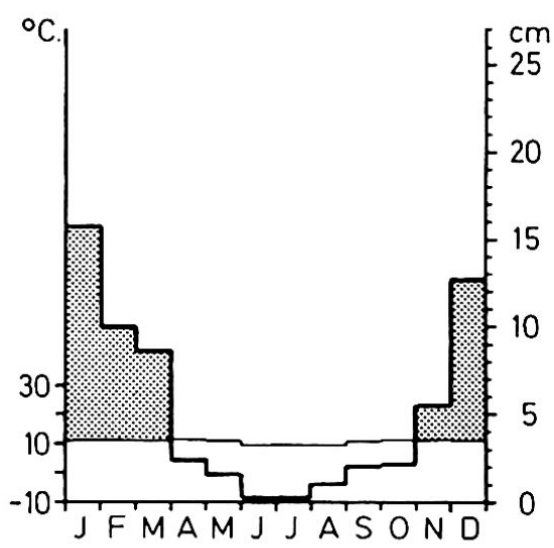

COCHABAMBA

$17^{\circ} 27^{\prime} \mathrm{S} / 66^{\circ} 6^{\prime} \mathrm{W}, 2550 \mathrm{~m}$

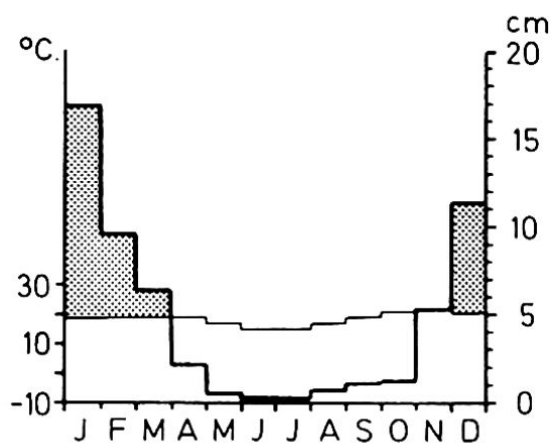

Fig. 2 Die sechs Klimadiagramme geben die humiden Monate gerastert wieder und veranschaulichen links semihumide und rechts semiaride Klimate der Anden. Untereinander stehen jeweils die Diagramme von zwei Stationen, die auch räumlich zusammengehören.

Quelle: ANUARIOS METEOROLÓGICOS 1974-1979 (Ulla Ulla nur 1975-1979). 
Mächtigkeit, der sich kaum vom Ort wegbewegt und, verglichen mit abfließendem Wasser, kaum meßbare Kalkmengen löst.

Temperaturmäßig liegen alle Monatsmittel nahe beim Jahresmittel. Dieses schwankt je nach Höhenlage der Station zwischen $4,4^{\circ} \mathrm{C}$ (Ulla Ulla, $4460 \mathrm{~m}$ ) und $18,3^{\circ} \mathrm{C}$ (Cochabamba, $2550 \mathrm{~m}$ ). Tiefe Temperaturen können die Karstprozesse vielfältig hemmen. Bei negativen Temperaturwerten fehlt der Wassernachschub in die Tiefe. Außerdem treten sehr viele Frostwechsel auf, d. h. daß die Temperatur häufig den Nullpunkt durchläuft (GRAF 1973, S. 135), und damit stellt sich ein ausgesprochenes Hochgebirgsklima mit intensiver Frostsprengung ein. Dabei wird nicht primär die Korrosion verhindert, denn sie geht bei zeitweiligen Wärmeschüben durchaus weiter, sondern die Formen werden sekundär durch Frostverwitterung zerstört. Ebenfalls in komplexer Art wirken die großen Tagesschwankungen der Temperatur, wie sie für die Tropen typisch sind (Tageszeitenklima nach TROLL 1959, S. 19-21). Berücksichtigt man die großen sonnen- und schattenseitigen Temperaturunterschie$\mathrm{de}^{5}$, so ergeben sich enorme effektive tageszeitliche Schwankungen. Damit ändern sich kurzzeitig der Sättigungsgrad des Wassers und seine Korrosionsfähigkeit. Gleichzeitig bewirken diese Gegensätze aber eine intensive Insolationsverwitterung, welche je nach den lithologischen Bedingungen Karren und Dolinen zurunden oder sogar zerstören kann.

Tiefe Temperaturen können sich auch positiv auf Karstprozesse auswirken, indem sie die Verdunstung vermindern und so das Wasserangebot steigern. Damit wird die effektive Feuchtzeit bzw. die karstaktive Zeit verlängert. Im ganzen gesehen, sind aber wohl im Untersuchungsraum sowohl die Niederschläge als auch die Temperaturen ungünstig für Karstprozesse. Kältemäßige Grenzbedingungen für den Karst liegen indessen nicht genau nachweisbar vor. Ein solcher Nachweis gestaltet sich schwierig, da ja das Klima oft nur indirekt limitiert: Die Grenze der Karstformung wäre erreicht, wo die Zerstörung der entstehenden Karstformen durch Verwitterung und Erosion ebenso schnell vorwärtsschreitet wie die Bildung der Formen. Karren und Dolinen sind aber oberhalb $4500 \mathrm{~m}$ gefunden worden, in einer Höhe, die dem Einsetzen von Frostbodenformen in den bolivianischen Anden entspricht (Untergrenze der Solifluktionsformen, GRAF 1973, S. 139). Also scheint die effektive Kältegrenze für Karst erst in der Nähe der Gletscherfirngebiete zu liegen, d.h. in der nivalen Stufe oberhalb rund $5200 \mathrm{~m}$. Den unmittelbaren Bereich der Gletscherzungen zwischen ca. 4800 und $5200 \mathrm{~m}$ muß man allerdings ausnehmen, weil hier allfällige Karstformen sekundär durch die Glazialerosion weggeputzt werden.

In ähnlicher Art wie das Klima können auch die petrographischen Voraussetzungen Karstprozesse auslösen oder limitieren. Diese Grenzbedingungen sollen im folgenden Kapitel diskutiert werden.

\section{Angetroffene Karstgesteine}

In Bolivien findet man wenig Karstgegenden, weil die verkarstungsfähigen Sedimentgesteine, Kalk, Dolomit und Gips, gegenüber kristallinen und vulkanischen Gesteinen weit zurückstehen. Zur Hauptsache sind die bolivianischen Anden nämlich aus paläozoischen Metamorphiten und tertiären Intrusivgesteinen und Vulkaniten aufgebaut. Verkarstungsfähige Gesteine treten einigermaßen häufig im Perm auf (Formationen Copacabana und Tiquina nach CABRERA \& PETERSEN 1936, zit. in PAREJA et al. 1978, S. 10). Aus der Trias existieren an vereinzelten Stellen in den südbolivianischen Anden ab ca. $19^{\circ} \mathrm{S}$ Kalke und Dolomite (Formation Vitiacua nach MATHER 1922, zit. in PAREJA et al. 1978, S. 11). Weitere Vorkommen entstammen erst v. a. der Kreidezeit. Einen Felsblock mit Karren aus kretazischem Gips zeigt Abb.6. Aus kretazischem Kalk besteht ein Karrenfeld auf einer nordostexponierten Felsterrasse $25 \mathrm{~km}$ südwestlich

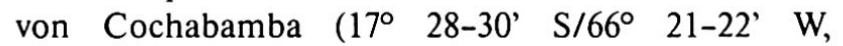
2800-3100 m). Er gehört zur Formation El Molino (LOHMANN \& BRANISA 1962, zit. in PAREJA et al. 1978, S. 12; GEOBOL, unpublizierte Aufnahme 1:50000 zur geologischen Karte Boliviens, Blatt 6341). Ebenfalls kretazisch sind zwei Gipsvorkommen ca. $10 \mathrm{~km}$ nördlich des Stadtrandes von $\mathrm{La} \mathrm{Paz}\left(16^{\circ} 22^{\prime} \mathrm{S} / 68^{\circ} 10^{\prime}\right.$ W und $16^{\circ} 24^{\prime}$ S/ $68^{\circ} 6^{\prime}$ 'W, 4500 m; Geol. Karte Boliviens $1: 100000$, Blatt 5944). Sie werden in Gruben abgebaut und dienen der Stukaturherstellung. Auch aus dem Tertiär finden sich vereinzelte Vorkommen von Kalk und Gips.

Auffällig ist, wie häufig und intensiv Karstprozesse im Gips ablaufen. Die Vermutung, daß Gips in Bolivien besonders anfällig auf eine Verkarstung reagiert, läßt sich allerdings nur mit einigen Indizien erhärten. Der häufigste Karstprozeß ist dabei nach zöTL (1974, S. 9/10) ein physikalischer Vorgang, indem sich der weniger wasserlösliche Anhydrit im Kontakt mit Wasser in gut löslichen Gips umwandelt:

$$
\mathrm{CaSO}_{4}+2 \mathrm{H}_{2} \mathrm{O} \rightleftarrows\left(\mathrm{CaSO}_{4} \cdot 2 \mathrm{H}_{2} \mathrm{O}\right)
$$

Der Gips kann also in der Regenzeit wegen seiner hohen Löslichkeit problemlos weggelöst werden. Zudem ist im bolivianischen Hochland oft der Salzgehalt groß, was durch die intensive Verdunstung oder auch geologisch bedingt sein kann. Man findet etliche Salz- und Gipslager in Diapiren (LOHMANN \& BRANISA 1962, zit. in PAREJA et al. 1978, S. 12), und zwar im südlichen Altiplano aus der Kreidezeit und im nördlichen aus dem Tertiär. Wegen der Anwesenheit von Fremdionen ist nun aber Gips in salzhaltigem Wasser besonders gut löslich (вÖGLI 1978, S. 34f.), was sich in verstärkter Gipslösung äußert. Auch die noch heute andauernde tektonische Aktivität der bolivianischen Anden fördert sicher den Gipskarst. So werden nämlich Hohlräume bereitgestellt, wo das Wasser zirkulieren und abfließen kann. 
All diesen verstärkenden Faktoren muß nun doch entgegengehalten werden, daß dem Gipskarst Grenzen in der Eindringtiefe gesetzt sind. Die Umwandlung von Anhydrit in Gips ist nämlich mit einer Volumenvermehrung auf das 1,557 fache verbunden (MOYE 1906, zit. in ZÖTL 1974, S. 10). Dadurch verstopfen Klüfte bald, und es kann sich kein weitgespanntes und in größere Tiefen reichendes Karstwassersystem ausbilden. Karstprozesse wirken in Gips also nur oberflächennah bis in maximal einige Meter Tiefe im Normalfall und stellen relativ kurzfristige Vorgänge dar. Im weiteren können Lösungsprozesse durch die starke Verdunstung in den semiariden Tropen ganz unterbunden oder gehemmt werden. Sie führt unmittelbar dazu, daß eine Gipslösung übersättigt wird und sich Gips ausscheidet. Besonders auch beim Gips wirken die großen tageszeitlichen Temperaturgegensätze der Erhaltung von Karstformen entgegen, da sie ausgesprochen häufig Abtragungsprozesse auslösen.

Einen Spezialfall gilt es abschließend zu erwähnen. In gerader Linie sind auf einer Gesamtdistanz von $1 \mathrm{~km}$ sechs Seelein am nördlichen Stadtrand von $\mathrm{La} \mathrm{Paz}$ angeordnet $\left(16^{\circ} 26^{\prime} \mathrm{S} / 68^{\circ} 9^{\prime} \mathrm{W}, 4250 \mathrm{~m}\right)$. Ihre trichterartige Form weist auf Karstprozesse hin. Auch die nahen Vorkommen von Gipsgruben, nur $5-6 \mathrm{~km}$ weiter im NW und NE, erhärten die Vermutung, daß es sich bei der Seenreihe um ein geologisch vorgezeichnetes Gipskarstphänomen handelt (mdl. Mitt. H.H.LOHMANN). Eine Parallelerscheinung dieser Art findet sich im Pacos-Valley in New Mexico/USA $\left(32^{\circ}\right.$ $10^{\prime} \mathrm{N} / 104^{\circ} 40^{\prime} \mathrm{W}$, mdl. Mitt. A. BÖGLI).

\section{Datierungsmöglichkeiten}

\subsection{Zum Alter von Karstquellen}

Das Tal, welches nach Charazani hinunterzieht, weist viele Hangquellen auf. Sie entspringen in $45^{\circ}$ gegen NW einfallenden Kalk- und Tonschieferschichten und sind teilweise als Schichtgrenzquellen anzusprechen (Abb. 7). Verschiedene Feldbeobachtungen legen aber den Schluß nahe, daß sie ihr Wasser auch aus höhergelegenen Karstflächen beziehen können und somit Karstquellen sind. Eine erste Beobachtung stützt sich auf die außerordentliche Häufigkeit dieser Quellen. Es sind zahlreiche weitere Hangquellen im W und NW von Huancarani in ähnlicher Situation auf $4500-4600 \mathrm{~m}$ gefunden worden $\left(15^{\circ} 17^{\prime} 30^{\prime \prime}\right.$ bis $15^{\circ} 18^{\prime}$ S $/ 69^{\circ} 0^{\prime}$ bis $69^{\circ} 2^{\prime}$ W). Sie haben meistens Anlaß zur Bildung kleiner Hangmoore gegeben. Dieser edaphischen Begünstigung der Vegetation widmet sich eine zweite Beobachtung. Normalerweise enthält die in der Puna verbreitete Steppenvegetation auf dieser Höhenstufe dominante Büschelgräser und daneben dickwurzlige Compositenkräuter (Senecio, Werneria), Polsterpflanzen (Pycnophyllum/Caryophyllaceen) sowie
Kleinsträucher (Tetraglochin strictum/Rosaceen, Baccharis/Compositen). Hingegen wachsen in Mooren dominant Distichia muscoides und daneben weitere Juncaceen (Juncus, Luzula), Cyperaceen (Carex), Gentiana sedifolia, Rosaceen (Alchemilla pinnata), Verbena minima sowie weiterhin Werneria pygmaea, Pycnophyllum molle und Gramineen (z. B. Aciachne pulvinata). Die Hangmoore setzen klare pflanzensoziologische Akzente in einer Landschaft, die sonst durchwegs von monotoner, an die Trockenheit angepaßter Vegetation besetzt ist. Es ist als hydrographische Besonderheit zu werten, daß etliche dieser Hangquellen ganzjährlich Wasser führen, also sehr wahrscheinlich Karstquellen sind.

Die Hangmoore enthalten bloß $20-35 \mathrm{~cm}$ mächtigen Torf. Davon ist eine Basisprobe ${ }^{14} \mathrm{C}$-datiert worden, die rezentes bis allenfalls um 100 Jahre Alter ergeben hat (UZ-136, $15^{\circ} 17^{\prime} \mathrm{S} / 69^{\circ}$ 1' $\mathrm{W}, 4580 \mathrm{~m}$ SW-Exposition). Damit zeigt es sich, daß die Karstentwässerung in der Umgebung des moorigen Standortes kaum viel älter sein kann. Die Ansiedlung einer spezifischen Moorvegetation weist allerdings darauf hin, daß sie doch mindestens einige Jahrhunderte zurückreichen muß. Die datierte Probe gibt nur ein

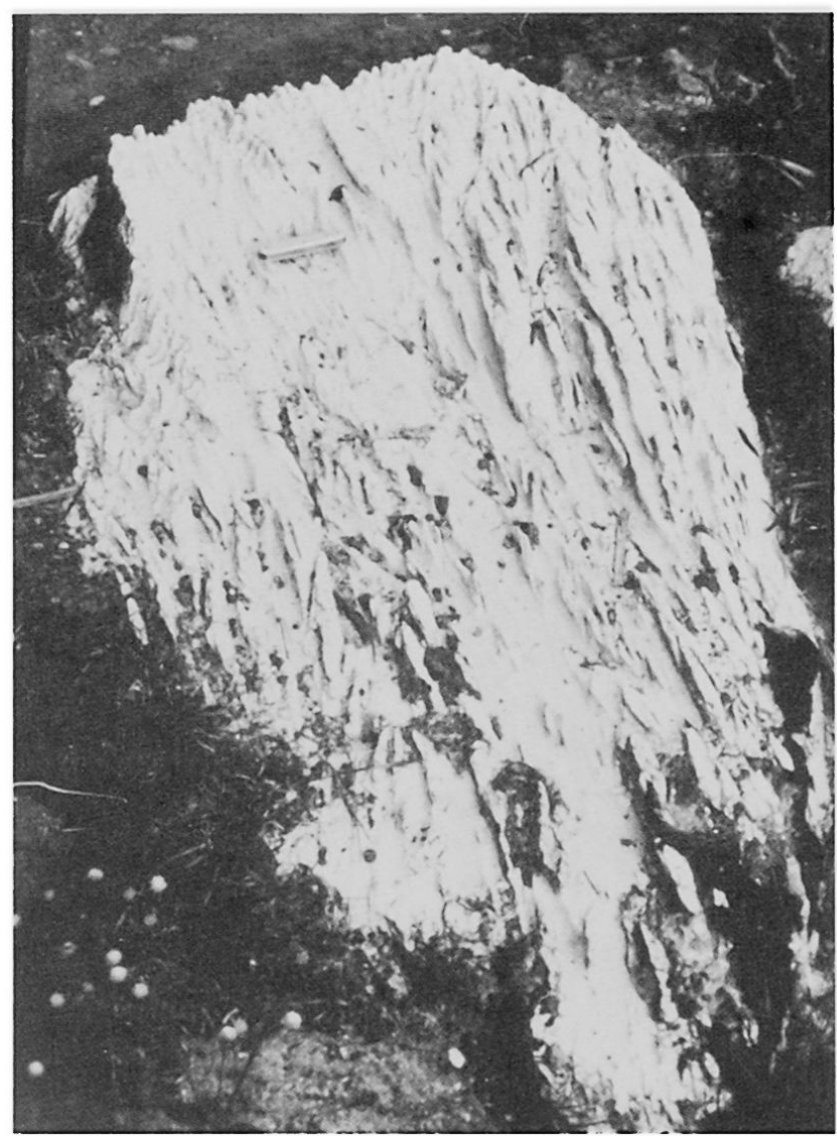

Abb. 6 Gipsblock außerhalb der Höhle San Pedro bei Sorata $\left(15^{\circ} 44^{\prime} \mathrm{S} / 68^{\circ} 41^{\prime} \mathrm{W}, 2400 \mathrm{~m}\right)$. Er trägt in typischer Art Karren, die auf eine intensive Gipslösung zurückgehen. 


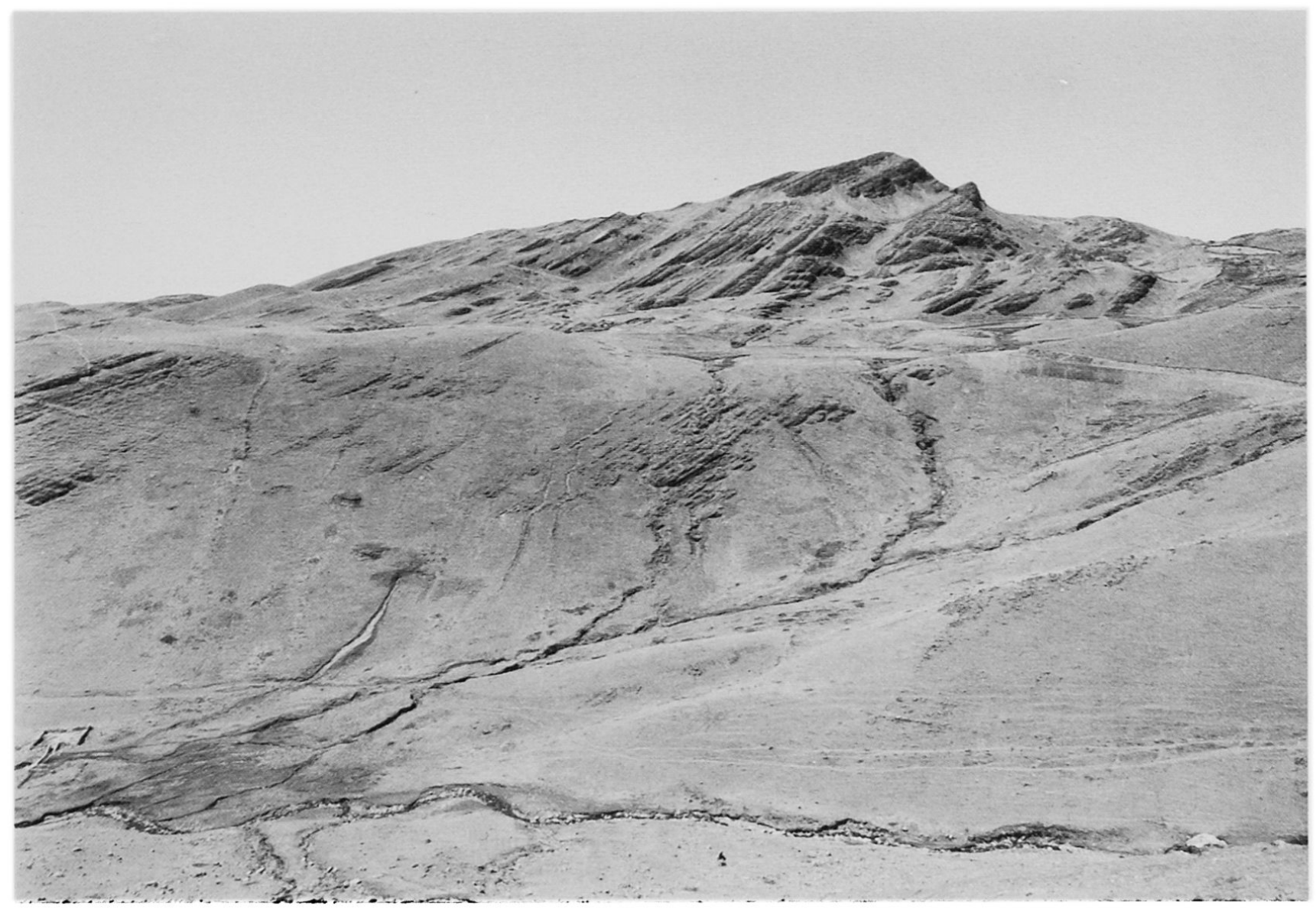

Abb. 7 Das Bild ist von W oberhalb Charazani aufgenommen und vermittelt einen Eindruck von der rechten Talseite (15० $12^{\prime} 40^{\prime \prime}$ $\mathrm{S} / 69^{\circ} 2^{\prime} \mathrm{W}$ ). Man erkennt in der Bildmitte und etwas links davon Quellen auf ungefähr halber Höhe zwischen der Sohle des Haupttals auf $4100 \mathrm{~m}$ und einer Hangterrasse auf ca. $4300 \mathrm{~m}$. Die Terrasse ist vermoort und weist mehrere (hier nicht sichtbare) Versickerungsstellen auf.

Minimalalter, und daher wäre ein hoher Wert natürlich aussagekräftiger gewesen. Immerhin kann mit dem geringen Torfalter ein Indiz dafür gewonnen werden, daß sich die heutige Entwässerung im Raum Huancarani/Charazani erst in jüngerer Zeit entwickelt hat und sich sehr wahrscheinlich noch ausweiten wird. Ich habe zudem versucht, das Minimalalter mit einem pollenanalytischen Profil abzustecken. Eine Abschätzung des Alters wäre in Anlehnung an das 9500 Jahre zurückreichende Pollenprofil F (GRAF 1981, S. 358) wohl möglich gewesen, da es aus einem Moor knapp $20 \mathrm{~km}$ nordwestlich von Huancarani stammt. Der schlechte Erhaltungszustand und die geringe Pollenfrequenz der Proben erlaubten aber keine Auszählung. Auch die Eintiefung der Gerinne kann in die Betrachtung einbezogen werden, denn sie hilft, das Alter der Erosion abzuschätzen. Die anstehenden Gesteine, Kalk und Tonschiefer, sind tektonisch wenig zerrüttet, so daß man annehmen muß, daß das Einschneiden der Bäche eher langsam erfolgt ist. Dennoch deutet die geringe Tiefe der Bachbetten auch auf ein kleines Alter hin.

\subsection{Zum Alter von Karren- und Dolinenfeldern}

Eine Lokalität wird nun beleuchtet, worin sich sowohl glaziale als auch karstbedingte Formungsprozesse niedergeschlagen haben. Im Quellgebiet des Río Charazani befindet sich ein leicht gewellter, ca. $1 \mathrm{~km}$ breiter Talabschnitt. Primär handelt es sich um eine Grundmoränenlandschaft, die von Gletschern aus der Kordillere von Apolobamba während der letzten Eiszeit geformt worden ist (LAUER 1979, S. 10; SEIBERT 1982, S. 150). Vereinzelte $2 \mathrm{~m}$ hohe Wallmoränen unmittelbar am Flußufer enthalten eindeutig gekritzte Sandsteine (15० 6' $\left.30^{\prime \prime} \mathrm{S} / 69^{\circ} 8^{\prime} \mathrm{W}, 4300 \mathrm{~m}\right)$. Die verstreuten kleinen Wannen sind mit Kalksteinen übersät, welche Karren tragen. Etliche Wannen sind wohl periodisch wassergefüllt, haben aber in der Trockenzeit im August 1977 dolinenartig ausgesehen. Da der Untergrund von permischen Kalken aufgebaut wird, ist eine wenn auch lokal eingeschränkte Karstformung und -hydrographie möglich. Indessen gilt für alle im Untersuchungsraum festgestellten Dolinen, daß sie sich im Gebiet befinden, das letzteiszeitlich 
von Gletschern bedeckt war. Aus all diesen Feststellungen heraus läßt sich vorläufig schließen, daß hier ähnliche Bildungsbedingungen und Altersverhältnisse wie beim hochalpinen Karst vorliegen. Nach dem letzteiszeitlichen Eisschliff und der Moränendeposition wurde die Oberfläche freigegeben, worauf eine Korrosion einsetzte. Der Eisrückzug erfolgte hier allgemein rasch und definitiv. Auf dem glazial vorgeformten Relief hat sich also frühestens 10000 Jahre BP (GRAF 1981, S.366) langsam eine Verkarstung eingestellt. Damit kann man die Dolinen in Nordbolivien als zumindest vorwiegend postglaziale Bildungen bezeichnen. Diese Folgerungen sind höchstens einzuschränken, wenn man die Dauer des Postglazials als zu kurz für die Schaffung gewisser Karstformen betrachtet. Dahin zielen vor allem Überlegungen zur Geschwindigkeit der Karrenbildung. Im Beispiel der Karren von Abb. 1 und 2 aus Pacotallani ist es wohl angebracht, die initiale Anlage von Klüften ins Tertiär und das Einsetzen der Karrenbildung bereits ins Pleistozän zu legen. Aber auch in diesem Fall hat eine glaziale Überprägung stattgefunden. Das belegen der Rundhöcker von Abb. 1 und der Moränenschutt von Abb. 8. Diese gekritzten Steine liegen auf einem nahegelegenen Kalkrücken, eingebettet in eine $20-40 \mathrm{~cm}$ mächtige Füllung von Karren. Nach dem Gletscherrückzug hat sich die endgültige Karstformung vollzogen. Erst seit 10000 Jahren haben sich die Kluft- und Rinnenkarren in ihrer heutigen Form ausgeprägt, aber ihre primäre Anlage reicht ins Pleistozän zurück.

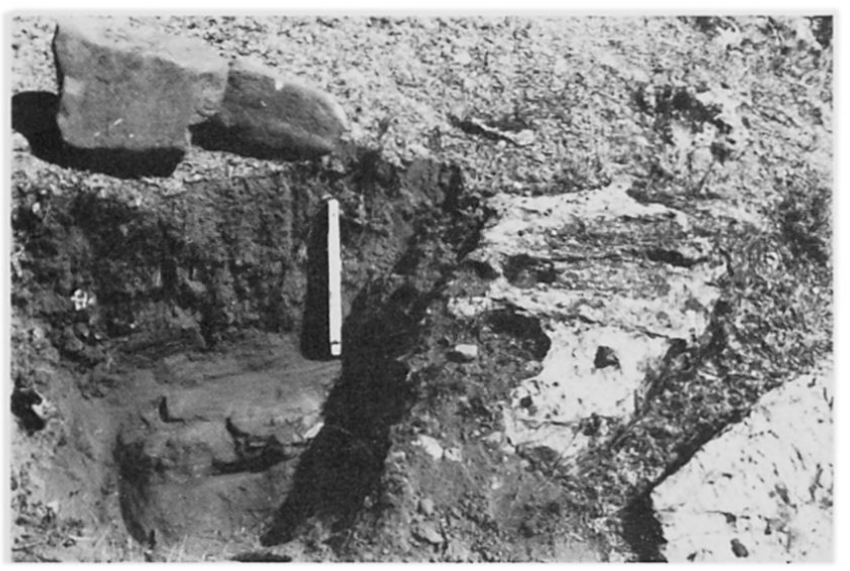

Abb. $8 \quad 10-20 \mathrm{~cm}$ breite Kluftkarren mustern einen Felsrücken bei Pacotallani ( $15^{\circ} 3^{\prime} 40^{\prime \prime} \mathrm{S} / 69^{\circ} 7^{\prime} \mathrm{W}, 4300 \mathrm{~m}$ ). Der ca. $30 \mathrm{~cm}$ mächtige Boden enthält Moränenschutt und ist in der linken Bildhälfte aufgegraben worden. Als Beispiel sind zwei gekritzte Steine oben hingelegt.

\section{Schlußfolgerungen}

Generell ist festzuhalten, $\mathrm{da} ß$ in Bolivien nur wenige Karstformen auftreten. Begrenzend wirkt der enge klimatische Spielraum, der in diesem Abschnitt der
Anden einerseits durch die saisonale Trockenheit und anderseits durch die ganzjährlich tiefen Mitteltemperaturen abgesteckt wird. Es fehlen ausgesprochene Großformen der heißen, \pm immerfeuchten Tropen wie der Kegelkarst, Poljen und ausgedehnte Höhlensysteme. Hingegen findet man Dolinen, kleine Karstquellen und Höhlen sowie Karrenfelder. Sie sind stark an geologische und tektonische Gegebenheiten geknüpft, weniger an klimatische Besonderheiten. Dolinen und ganze Dolinenfelder treten besonders in leicht verwitternden Karstgesteinen auf, z. B. in Gips sowie tektonisch zerrütteten oder mergeligen, dünnbankigen Kalken. Bei den Karren spielen neben den petrographischen Voraussetzungen auch die Topographie und die Art der anfallenden Niederschläge entscheidend mit, was mit einem Beispiel von Copacabana in Kap. 1 veranschaulicht worden ist.

Den wichtigsten limitierenden Faktor stellt das Gestein dar. Besonders aber bei Gips geschieht in Bolivien, wie überall, eine intensive Verkarstung. Auch in zum Teil silikatischem Gestein sind karstähnliche Lösungsvorgänge und Karrenbildungen festgestellt worden (Pseudokarren nach BöGLI 1978, S. 61). Einschränkend ist aber zuzugeben, da $ß$ Karstphänomene wegen der eingeschränkten Zahl von Kalk- und Gipsgebieten in Bolivien wenig verbreitet sind. Sie bringen indessen, gemessen an den klimatischen Bedingungen, eine bemerkenswerte Formausgestaltung hervor.

Das zeitliche Einordnen der Karstprozesse ist schwierig. Datierung und vergleichende glazialmorphologische Feldbefunde lassen aber doch annehmen, daß die Karren in den bolivianischen Anden zur Hauptsache postglazial sind, aber stark eingetiefte Exemplare wohl ihren Ursprung im Pleistozän haben. Auch die Dolinen stellen häufig Weiterentwicklungen von eiszeitlich entstandenen Anlagen dar. Hingegen fältt die Bildung allfälliger Karstquellen eindeutig ins Postglazial.

\section{Summary}

We studied karst forms in the Bolivian Highland. There are clints (or lapiés, karrens, see photos 1, 2 and 3 ), dolines (photos 4,5 and 8), caverns and karst springs (photo 7), but no poljes and conical karst. They can be formed in spite of extreme climatic conditions, namely low temperatures in altitudes of up to $4600 \mathrm{~m}$ and sometimes only four humid months. In respect to the petrography we note a relatively wide range: limestone, calcareous sandstone, dolomite and especially gypsum (photo 6) are intensely affected by corrosion. All the dolines and clints found in the Andes are mainly postglacial, but were preformed in the Pleistocene. 


\section{Resumen}

Se han estudiado los modelados cársicos en los Andes bolivianos, entre ellos lenares (ó lapiaz, ver láminas 1, 2 y 3), dolinas (láminas 4,5 y 8), grutas y fuentes (lámina 7) que están formados aún con condiciones climáticas extremas, con temperaturas bajas en altitudes de hasta de más de $4600 \mathrm{msnm}$ y a veces con sólo 4 meses húmedos. Faltan poljés y carso de torrecillas (turm-karsts). Respecto a la petrografia se nota una variedad relativamente grande: caliza, arenisca calcárea, dolomía y especialmente yeso (lámina 6) están afectados intensamente por la corrosión. Las dolinas y los lenares encontrados en los Andes han sido formados sobre todo en el período postglacial, pero estaban preformados en el Pleistoceno.

\section{Literatur}

ANUARIOS METEOROLÓGICOS (1974-1979): Klimajahrbücher des Servicio Nacional de Meteorologia e Hidrologia, La Paz - Bolivia, je ca. $110 \mathrm{~S}$.

BIESE, W. (1931): Über Höhlenbildung, I. Teil. Entstehung der Gipshöhlen am südlichen Harzrand und am Kyffhäuser. In: Abh. Preuß. Geol. Landesanstalt, N. F. 37, 1-71.

BÖGLI, A. (1951): Probleme der Karrenbildung. In: Geographica Helvetica 6/3, 191-204.

- (1972): Gips in Höhlen. In: Urner Mineralienfreund 10/6, $77-84$.

- (1975): Die wichtigsten Karrenformen der Kalkalpen. In: GAMS, I. (ed.), Karst processes and relevant landforms, University of Ljubljana, 141-149.

- (1978): Karsthydrographie und physische Speläologie, Springer-Verlag Berlin, 1-278.

CORBEL, J. (1959): Karsts du Yucatan et de la Floride. In: Bull. Ass. géographes français 282/283, 2-14.

GERSTENHAUER, A. (1966): Beiträge zur Geomorphologie des mittleren und nördlichen Chiapas (Mexico) unter besonderer Berücksichtigung des Karstformenschatzes. In: Frankfurter Geogr. H. 41, 1-91.

GRAF, K. (1973): Vergleichende Betrachtungen zur Solifluktion in verschiedenen Breitenlagen. In: Z. Geomorph. N. F., Suppl.Band 16, 104-154.

- (1981): Palynological investigations of two post-glacial peat bogs near the boundary of Bolivia and Peru. In: Journal of Biogeogr. 8, 353-368.

KINZL, H. (1951): Karsterscheinungen in den peruanischen Anden. In: Festschrift J. Sölch, Wien, 52-58.

LAUER, W. (1960): Klimadiagramme. In: Erdkunde XIV, 3 , 232-242.

- (1979): Im Vorland der Apolobamba-Kordillere. In: Coll. Inst. Anthr. 21, St. Augustin, 9-15.

LEHMANN, H. (1954): Der tropische Kegelkarst auf den Großen Antillen. In: Erdkunde 8/1, 130-139.

MAROCCO, R. (1974): Estudio geológico de la región entre Cuzco y Machu Picchu. In: Bull. Inst. français d'études andines III/2,1-27.
MIOTKE, F.-D. (1973): Die Tieferlegung der Oberflächen zwischen Mogoten in Puerto Rico (östlich Arecibo). In: Geogr. Ztschr. Hettner, Beiheft 32, 34-43.

MONROE, W. H. (1960): Sinkholes and Towers in the KarstArea of Northcentral Puerto Rico. In: Geol. Survey Prof. Papers 400-B, 356-360.

NUÑEZ JIMENEZ, A. et al. (1969): Typen des tropischen Karstes auf Kuba. Přirodovědné práce ústavủ Československé akademie věd Acta Sc. Nat. Brno III, N. Series 11, 1-45.

PAREJA, J. et al. (1978): Mapa geológico de Bolivia $1: 1000$ 000. YPFB \& GEOBOL, La Paz, 1-27 (Begleittext zur Karte).

PEREZ G., H. (1972): Contribución al conocimiento geológico de la zona de Ulla Ulla - Charazani. Tesis de grado UMSA 106, ca. $80 \mathrm{~S}$.

PFEFFER, K.-H. (1976): Probleme der Genese von Oberflächenformen auf Kalkgestein. In: Ztschr. Geomorph. N. F., Suppl.-Band 26, 6-34.

SEIBERT, P. (1982): Ökosystemforschung in den bolivianischen Anden. In: Naturwiss. Rdsch. 35/4, 147-157.

SWEETING, M.M. (1972): Karst landforms. The Macmillan Press Ltd., London, 1-362.

TROLL, C. (1959): Die tropischen Gebirge. Ihre dreidimensionale klimatische und pflanzengeographische Zonierung. In: Bonner Geogr. Abh. 25, 1-93.

VERSEY, H.R. (1972): Karst of Jamaica. In: HERAK, M. \& STRINGFIELD, V.T., Karst. Elsevier Publ. Comp., Amsterdam, 445-466.

WENZENS, G. (1973): Fossile und rezente Karstformen im semiariden Bereich der Sierra Madre Oriental (Nordmexico). In: Geogr. Ztschr. Hettner, Beiheft 32: 54-69.

ZÖTL, J. G. (1974): Karsthydrogeologie. Springer-Verlag, Wien, 1-291.

\section{Anmerkungen}

'Für die Durchsicht des Manuskripts danke ich meinem Kollegen und Lehrer, Prof. Dr. A. BÖGLI.

${ }^{2}$ Letzteiszeitlich haben Gletscherzungen auf dieser Halbinsel sicher bis $4050 \mathrm{~m}$ hinabgereicht (GRAF 1981, S. 11), hingegen waren hier in den letzten 10000 Jahren überhaupt keine Gletscher mehr vorhanden. Die Klimaänderungen hielten sich also im Postglazial sehr in Grenzen.

${ }^{3}$ Ein schön ausgebildeter Karrentyp, nämlich Wandkarren, ist in Peru festgestellt worden, einige $\mathrm{km}$ nordöstlich von Cuzco bei Kenko ( $13^{\circ} 26^{\prime} \mathrm{S} / 71^{\circ} 55^{\prime} \mathrm{W}, 3500 \mathrm{~m}$ ). Beim Gestein handelt es sich um kretazischen Kalk nach MAROCCO (1974, Kartenbeilage). KINZL (1951, S. 53) hat in dieser eindrücklichen Karstlokalität neben Karrenfeldern auch Höhlen gefunden.

${ }^{4}$ Primär ist Kalk i.d.R. schlechter bewachsen als Gips, da im Gips mehr unlösliche Verunreinigungen vorliegen. Da aber Gips geomorphologisch sehr weich ist und hier nur eine schüttere Steppenvegetation wächst, kommt der Boden an Hängen leicht ins Rutschen und reißt auf, so daß sich Badlands entwickeln können.

${ }^{5}$ Eine stichprobenhafte Temperaturmessung ergab im Nov. 1974 in La Paz um die Mittagszeit $18^{\circ} \mathrm{C}$ im Schatten und $40^{\circ} \mathrm{C}$ unmittelbar daneben an der Sonne. 